\title{
Solution of System of Generalized Abel's Integral Equation using Gegenbauer Wavelets
}

\author{
Ashish Pathak \\ Department of Mathematics \& Statistics \\ Dr. Harisingh Gour Central University \\ Sagar-470003, India
}

\author{
Rajeev Kr. Singh \\ Department of Mathematics \\ Sikkim Manipal Institute of Technology \\ Sikkim-737136, India
}

\begin{abstract}
This has been seen that in some application for finding the analytical solution of mathematical problem is too complicated, so in recent years researcher has devoted lots of afforts to find the numerical solution of the equation. In this paper an application of the Gegenbauer wavelet method is applied to solve system of generalized Abel's integral equation. This wavelet reduces the system of generalized Abel's integral equation to a system of linear equation in generalized case such that the solution of the resulting system gives the unknown Gegenbauer wavelet coefficient of the solutions. Illustrative examples have been provided to demonstrate the validity and applicability of the proposed method and result has been compaired with the exact solution. Lastly we have shown the error analysis to the proposed method and found that it is an quite efficient and has high accuracy.
\end{abstract}

\section{Keywords:}

Generalized Abel's integral equation; Gegenbauer polynomial; Gegenbauer wavelets.

\section{INTRODUCTION}

As a matter of fact it is said that many engineering and applied science problem like water waves, potential theory and electrostatics are reduced to solve integral equations. Since these equation we can not solve exactly so we require to obtain approximate solutions. In this paper we have proposed to solve system of generalized Abel's integral equation. So far there are numerous numerical methods which have been focusing on the solution of generalized Abel's integral equation like for exam Lowengrub and Waltson[1] this method in which the system has been solved by reducing the system to an equivalent coupled Riemann- Hilbert boundary value problems, Waltson[2] develop a method for solving certain systems of generalized Abel's integral equations by constructing an equivalent singular integral equation , Mandal solve the system for $\alpha=1, \mu=\frac{1}{2}, u_{i j}(x)=1$ by using fractional calculus[4], Panday and Mandal [3] solve this system by using Bernstien polynomial. Recently Ashish and Upreti [5] has solve this system of generalized Abel's integral equation by using Legendre multi-wavelets.

In the present paper we present a new approach for evaluating the approximate solution of the system (1)expanding the unknown function $f_{i}(x), i=1,2$ in terms of Gegenbauer wavelet [6] in $L_{w^{\alpha}}^{2}(0,1]$ space. We can reduce these system into system of lin- ear equations for given $h_{i}(x), i=1,2$. An error estimation of the system is discuss and some examples are given to show the efficiency and accuracy of the present method.

Consider the system of generalized Abel's integral equation[5]

$u_{11}(x) \int_{0}^{x} \frac{f_{1}(t)}{\left(x^{n}-t^{n}\right)^{\mu}} d t+u_{12}(x) \int_{0}^{1} \frac{f_{2}(t)}{\left(t^{n}-x^{n}\right)^{\mu}} d t=h_{1}(x)$
$u_{21}(x) \int_{x}^{1} \frac{f_{1}(t)}{\left(t^{n}-x^{n}\right)^{\mu}} d t+u_{22}(x) \int_{0}^{x} \frac{f_{2}(t)}{\left(x^{n}-t^{n}\right)^{\mu}} d t=h_{2}(x)$,

where $x \in(0,1), 0<\mu<1, n=1,2$ and $u_{i j}(x), i, j=1,2$ are continuous on $[0,1]$.

\section{GEGENBAUER WAVELETS (G W) AND ITS PROPERTIES}

Wavelet constitute a family of functions constructed by performing translation and dilation on a single function $\psi($.$) , where \psi($.$) is a$ mother wavelet. We define family of continuous wavelets [7] as

$$
\psi_{a, b}(\bullet)=\frac{1}{\sqrt{|a|}} \psi\left(\frac{\bullet-b}{a}\right) \quad a, b \in R, a \neq 0,
$$

where $a$ is called scaling parameter and $b$ is translation parameter. By discretization of [7] these parameters $a=2^{-k}$ and $b=n 2^{-k}$, we have

$$
\psi_{k, n}(\bullet)=2^{\frac{k}{2}} \psi\left(2^{k} \bullet-n\right) \quad k, n \in Z,
$$

where $\psi_{k, n}($.$) forms an orthonormal basis for L^{2}(R)$.

Gegenbauer wavelets $(\mathrm{G} \mathrm{W}) \psi_{n m}^{\alpha}(x)=\psi(k, n, m, \alpha, x)$ have five parameters; translation parameter $n=1 \ldots 2^{k-1}$, dilation parameter $k$ can assume any positive integer, $m$ is the order of Gegenbauer polynomial , $x$ is the normalized time [7] and last parameter $\alpha>$ $-\frac{1}{2}$. They are defined on $[0,1)$ as following form

$$
\psi_{n m}^{\alpha}(x)=\left\{\begin{array}{ccc}
2^{k / 2} \eta_{m}^{\alpha} \mathbf{G}_{m}^{\alpha}\left(2^{k} x-2 n+1\right), & \text { for } & \frac{n-1}{2^{k-1}} \leq x<\frac{n}{2^{k-1}}, \\
0, & \text { otherwise }
\end{array}\right\} \text {, }
$$

where $m=0,1,2, \ldots, M-1$, in eq(4) $\eta_{m}^{\alpha}=\sqrt{\frac{m !(m+\alpha)(\Gamma(\alpha))^{2}}{\pi 2^{1-2 \alpha} \Gamma(m+2 \alpha)}}$ is used for orthonormality and $\mathbf{G}_{m}^{\alpha}(x)=C_{m}^{\alpha}(2 x-1)$. Here $C_{m}^{\alpha}(x), \alpha>-1 / 2$ are the Gegenbauer polynomials which are 
orthogonal with respect to the weight function $\tilde{\omega}^{\alpha}(x)=(1-$ $\left.x^{2}\right)^{\alpha-1 / 2}$ on the interval $[-1,1]$. They are defined on the interval $[-1,1]$ by the following way [8]

$C_{m}^{\alpha}(x)=\sum_{r=0}^{[m / 2]} \frac{(-1)^{r} \Gamma(m-r+\alpha)}{\Gamma(\alpha)(r) !(m-2 r) !}(2 x)^{m-2 r} \quad, m=0,1,2,3 \ldots$.

Some of Gegenbauer polynomials are

$$
\begin{aligned}
& C_{0}^{\alpha}(x)=1 \\
& C_{1}^{\alpha}(x)=2 \alpha x \\
& C_{2}^{\alpha}(x)=2 \alpha(1+\alpha) x^{2}-\alpha \\
& C_{3}^{\alpha}(x)=4 / 3 \alpha(1+\alpha)(2+\alpha) x^{3}-2 \alpha(1+\alpha) x
\end{aligned}
$$

In fact, Gegenbauer polynomials form a large family of orthogonal polynomials with the famous Chebyshev polynomials of the first kind, $T_{m}(x)$, Chebyshev polynomials of the second kind, $U_{m}(x)$, and the Legendre polynomials, $P_{m}(x)$, as its special cases and their relationships are given in [8].

We should note that in dealing with the Gegenbauer wavelets the weight function $\omega^{\alpha}(x)=\tilde{\omega}^{\alpha}(2 x-1)$ have to be dilated and translated as $\omega_{n}^{\alpha}(x)=\tilde{\omega}^{\alpha}\left(2^{k} x-2 n+1\right)$ to get orthogonal G W

\section{FUNCTION APPROXIMATION AND CONVERGENCE}

We define the space $L_{\omega^{\alpha}}^{2}(0,1)$ as

$$
L_{\omega^{\alpha}}^{2}(0,1)=\left\{f \mid f \text { is measurable \& }\|f\|_{L_{\omega^{\alpha}}^{2}}<\infty\right\}
$$

where $\|f\|_{L_{\omega^{\alpha}}^{2}}=\left(\int_{0}^{1}|f(x)|^{2} \omega^{\alpha}(x) d x\right)^{1 / 2}$ and $\tilde{\omega}^{\alpha}(x)=$ $\left(1-x^{2}\right)^{\alpha-1 / 2}$ for $\alpha>-1 / 2$.

In particular $L_{\omega^{\alpha}}^{2}(0,1)$ is Hilbert space with the following inner product and norm

$$
\langle f, g\rangle_{L_{\omega^{\alpha}}^{2}}=\int_{0}^{1} f(x) \overline{g(x)} \omega^{\alpha}(x) d x,\|f\|_{L_{\omega \alpha}^{2}}=\langle f, f\rangle_{L_{\omega}^{\alpha}}^{2} .
$$

A function $f(x) \in L_{\omega^{\alpha}}^{2}(0,1)$, may be expressed in terms of the $\mathrm{G}$ $\mathrm{W}$ as,

$$
f(x)=\sum_{n=1}^{\infty} \sum_{m \in Z} c_{n m}^{\alpha} \psi_{n m}^{\alpha}(x),
$$

where the coefficient $c_{n m}^{\alpha}$ are given by

$$
c_{n m}^{\alpha}=\left(f(x), \psi_{n m}^{\alpha}\right)_{w_{n}^{\alpha}}=\int_{0}^{1} w_{n}^{\alpha}(x) \psi_{n m}^{\alpha}(x) f(x) d x .
$$

We can approximate the function $f(x)$ by the truncated series

$$
f(x) \simeq \sum_{n=1}^{2^{k-1}} \sum_{m=0}^{M-1} c_{n m}^{\alpha} \psi_{n m}^{\alpha}(x)=\left[C^{\alpha}\right]^{T} \Psi^{\alpha}(x)
$$

where the coefficient vector $C^{\alpha}$ and $\mathrm{G} \mathrm{W}$ vector function $\Psi^{\alpha}(x)$ are given by

$C^{\alpha}=\left[c_{10}^{\alpha}, \ldots, c_{2^{k-1}(M-1)}^{\alpha}\right]^{T}$ and $\Psi^{\alpha}(x)=\left[\psi_{10}^{\alpha}, \ldots, \psi_{2^{k-1}(M-1)}^{\alpha}\right]^{T}$.

Now, its convergence is given by the theorem.

THEOREM 1. Let $f(x) \in L_{\omega^{\alpha}}^{2}(0,1)$ and whose second derivative bounded in $(0,1)$, say $\left|f^{\prime \prime}(x)\right|<\boldsymbol{M}$, can be expanded as an infinite sum of Gegenbauer wavelets and the series converges uniformly to the function $f(x)$, that is:

$$
f(x)=\sum_{n=1}^{\infty} \sum_{m=0}^{\infty} c_{n m}^{\alpha} \psi_{n m}^{\alpha}(x), \quad \forall \alpha>-1 / 2,
$$

where $c_{n m}^{\alpha}=\left(f(x), \psi_{n m}^{\alpha}(x)\right)$ and $(\cdot, \cdot)$ denote the inner product in $L_{\omega^{\alpha}}^{2}(0,1)$.

\section{Proof. [6]}

THEOREM 2. (Accuracy Estimation) Let $f(x) \in L_{\omega^{\alpha}}^{2}(0,1)$ with bounded second order derivative $\left|f^{\prime \prime}(x)\right| \leq M$ where $M>0$, then we have the following accuracy estimation

$$
\left(\delta_{n m}^{\alpha}\right)^{2}<M \sum_{n=2}^{\infty} \sum_{m=M}^{\infty} \frac{1}{n^{5} m(2 \alpha+m)(m-1)(1+2 \alpha+m)} \forall \alpha \in N,
$$

where

$$
\left(\delta_{n m}^{\alpha}\right)^{2}=\int_{0}^{1}\left[f(x)-\sum_{n=1}^{2^{k-1}} \sum_{m=0}^{M-1} c_{n m}^{\alpha} \psi_{n, m}^{\alpha} d x\right]^{2} \omega_{n}^{\alpha}(x) .
$$

PROOF. [6]

Using the Gegenbauer wavelets basis, we have three degree of freedom as dilation parameter $k$, number of basis $M$ and $\alpha$, which increases the accuracy of the new method.

\section{METHOD OF SOLUTION}

For finding approximate solution of $(1), f_{1}(t)$ and $f_{2}(t)$ are approximated by using (8)

$\left.f_{1}(t) \approx \tilde{f_{1}^{\alpha}}(t)=\left[C_{1}^{\alpha}\right]^{T} \psi^{\alpha}(t), \quad f_{2}(t) \approx \tilde{f_{2}^{\alpha}}(t)=\left[C_{2}^{\alpha}\right]^{T} \psi^{\alpha}(t) 11\right)$ where $\left[C_{1}^{\alpha}\right]^{T}$ and $\left[C_{2}^{\alpha}\right]^{T}$ are unknown constants to be determined. Then from (11) and (1) we get

$$
\begin{gathered}
u_{11}(x)\left[C_{1}^{\alpha}\right]^{T} \Psi_{1}^{\alpha}(x)+u_{12}(x)\left[C_{2}^{\alpha}\right]^{T} \Psi_{2}^{\alpha}(x)=h_{1}^{\alpha}(x), \\
u_{21}(x)\left[C_{1}^{\alpha}\right]^{T} \Psi_{2}^{\alpha}(x)+u_{22}(x)\left[C_{2}^{\alpha}\right]^{T} \Psi_{1}^{\alpha}(x)=h_{2}^{\alpha}(x),
\end{gathered}
$$

where for $\forall x \in(0,1)$

$$
\begin{aligned}
& \Psi_{1}^{\alpha}(x)=\int_{0}^{x} \frac{\psi^{\alpha}(t)}{\left(x^{n}-t^{n}\right)^{\mu}} d t \\
& \Psi_{2}^{\alpha}(x)=\int_{x}^{1} \frac{\psi^{\alpha}(t)}{\left(t^{n}-x^{n}\right)^{\mu}} d t .
\end{aligned}
$$

Now, by putting $x=x_{l}, l=0,1,2 \ldots,(M-1) 2^{k-1}$ in 12$)$, where $x_{l}$ 's are chosen as suitable distinct points in $(0,1)$ such that $0<x_{0}, x_{1}, \ldots, x_{(M-1) 2^{k-1}}<1$. We obtain the linear systems

$$
\begin{gathered}
u_{11}\left(x_{l}\right) C_{1}^{\alpha} \Psi_{1}^{\alpha}\left(x_{l}\right)+u_{12}\left(x_{l}\right) C_{2}^{\alpha} \Psi_{2}^{\alpha}\left(x_{l}\right)=h_{1}^{\alpha}\left(x_{l}\right), \\
u_{21}\left(x_{l}\right) C_{1}^{\alpha} \Psi_{2}^{\alpha}\left(x_{l}\right)+u_{22}\left(x_{l}\right) C_{2}^{\alpha} \Psi_{1}^{\alpha}\left(x_{l}\right)=h_{2}^{\alpha}\left(x_{l}\right)
\end{gathered}
$$


The linear systems (14) can be easily solved by any standard method for the unknown constants $C_{1}^{\alpha}$ and $C_{2}^{\alpha}$ provided of course the coefficient matrix is nonsingular. It is emphasized that it is always possible to choose distinct points $x_{l} \in(0,1)$ such that this is possible. The computed $C_{1}^{\alpha}$ and $C_{2}^{\alpha}$ are then used in (11) to obtained the approximate solution $\tilde{f_{1}^{\alpha}}(t)$ and $\tilde{f_{2}^{\alpha}}(t)$.

\section{ERROR ESTIMATION}

In this section an error estimation for the approximation of solution of (1) is discussed. Let us consider $e_{k n}^{1 \alpha}(t)=f_{1}(t)-\tilde{f}_{1}^{\alpha}(t)$ and $e_{k n}^{2 \alpha}(t)=f_{2}(t)-\tilde{f_{2}^{\alpha}}(t)$ as the error functions of the approximate solution $\tilde{f}_{i}^{\alpha}(t), i=(1,2)$ respectively, where $f_{i}(t), i=1,2$ are the exact solution of (1) and using approximate solution in (1), we get

$$
\begin{aligned}
h_{1}(x)+T_{k n}^{1 \alpha}(x) & =u_{11}(x) \int_{0}^{x} \frac{\tilde{f_{1}^{\alpha}}}{\left(x^{n}-t^{n}\right)^{\mu}} d t \\
& +u_{12}(x) \int_{x}^{1} \frac{\tilde{f_{2}^{\alpha}}}{\left(t^{n}-x^{n}\right)^{\mu}} d t \\
h_{2}(x)+T_{k n}^{2 \alpha}(x) & =u_{21}(x) \int_{x}^{1} \frac{\tilde{f_{1}^{\alpha}}}{\left(t^{n}-x^{n}\right)^{\mu}} d t \\
& +u_{22}(x) \int_{0}^{x} \frac{\tilde{f_{2}^{\alpha}}}{\left(x^{n}-t^{n}\right)^{\mu}} d t
\end{aligned}
$$

where $T_{k n}^{i \alpha}(x), i=1,2$ are small perturbation quantity.

We proceed to find an approximations $\tilde{e}_{k n}^{1 \alpha}(t)$ and $\tilde{e}_{k n}^{2 \alpha}(t)$ to the error of functions $e_{k n}^{1 \alpha}(t)$ and $e_{k n}^{2 \alpha}(t)$ respectively . Now, using (1) and (15) we obtain

$$
\begin{aligned}
-T_{k n}^{1 \alpha}(x) & =u_{11}(x) \int_{0}^{x} \frac{e_{k n}^{1 \alpha}(t)}{\left(x^{n}-t^{n}\right)^{\mu}} d t \\
& +u_{12}(x) \int_{x}^{1} \frac{e_{k n}^{2 \alpha}(t)}{\left(t^{n}-x^{n}\right)^{\mu}} d t \\
-T_{k n}^{2 \alpha}(x) & =u_{21}(x) \int_{x}^{1} \frac{e_{k n}^{1 \alpha}(t)}{\left(t^{n}-x^{n}\right)^{\mu}} d t \\
& +u_{22}(x) \int_{0}^{x} \frac{e_{k n}^{2 \alpha}(t)}{\left(x^{n}-t^{n}\right)^{\mu}} d t
\end{aligned}
$$

It should be noted that in order to construct the approximations $\tilde{e}_{k n}^{1 \alpha}(t)$ and $\tilde{e}_{k n}^{2 \alpha}(t)$ to the error functions $e_{k n}^{1 \alpha}(t)$ and $e_{k n}^{2 \alpha}(t)$ respectively, only equation 16 to recalculated in same way we did before for the solution of equation (1).

\section{ILLUSTRATIVE EXAMPLES}

EXAMPLE 1. Consider the system of generalized Abel's integral equation (1) with $u_{i, j}=1$ for $i, j=1,2, n=1$ and $\mu=\frac{1}{2}$ with

$h_{1}(x)=\frac{4}{3} x^{\frac{3}{2}}+\frac{2}{15} \sqrt{1-x}\left(1+2 x+8 x^{2}\right)+2$ Dawson $F[\sqrt{x}]$

$h_{2}(x)=\frac{16}{15} x^{\frac{5}{2}}+\frac{2}{3} \sqrt{1-x}(1+2 x)+e^{-x} \sqrt{\pi} \operatorname{Erf}[\sqrt{(1-x)}]$.

which has exact solutions $f_{1}(x)=x+e^{x}$ and $f_{2}(x)=x^{2}$. If we apply the Gegenbauer wavelet approach and solve given equation by taking $M=6, k=1$ and $\alpha=1 / 2$ we obtain the approximate solution and compare result with the exact solution, Gegenbauer wavelets solution for $x \in(0,1)$ which is show in the table 1 .

EXAMPLE 2. Consider the system of generalized Abel's integral equation (1) with $u_{i, j}=1$ for $i, j=1,2, n=1$ and $\mu=\frac{1}{2}$ with

$$
\begin{gathered}
h_{1}(x)=\frac{4}{3} x^{\frac{3}{2}}+\frac{2}{15} \sqrt{1-x}\left(3+4 x+8 x^{2}\right) \\
h_{2}(x)=\frac{16}{15} x^{\frac{5}{2}}+\frac{2}{3} \sqrt{1-x}(1+2 x) .
\end{gathered}
$$

which has exact solutions $f_{1}(x)=x$ and $f_{2}(x)=x^{2}$. If we apply the Gegenbauer wavelet approach and solve (14) by taking $M=$ $3, k=1$ and $\alpha>0$ we obtain the exact solution

$$
\begin{aligned}
x= & \frac{\pi^{1 / 4}}{2 \sqrt{2}} \sqrt{\frac{\Gamma\left[\frac{1}{2}+\alpha\right]}{\Gamma[1+\alpha]}} \psi_{0,0}^{\alpha}(x)+\frac{\sqrt{\pi}}{4 \alpha} \sqrt{\frac{4^{-\alpha} \Gamma[1+2 \alpha]}{(1+\alpha) \Gamma[\alpha]^{2}}} \psi_{0,1}^{\alpha}(x), \\
x^{2} & =\frac{\pi^{1 / 4}(3+2 \alpha)}{8 \sqrt{2}(1+\alpha)} \sqrt{\frac{\Gamma\left[\frac{1}{2}+\alpha\right]}{\Gamma[1+\alpha]}} \psi_{0,0}^{\alpha}(x)+\frac{\sqrt{\pi}}{4 \alpha} \sqrt{\frac{4^{-\alpha} \Gamma[1+2 \alpha]}{(1+\alpha) \Gamma[\alpha]^{2}}} \\
& \times \psi_{0,1}^{\alpha}(x)+\frac{\alpha \Gamma\left[\frac{3}{2}+\alpha\right]}{4 \Gamma[3+\alpha]} \sqrt{\frac{(2+\alpha) \Gamma[\alpha]^{2}}{2^{1-2 \alpha} \Gamma[2+2 \alpha]}} \psi_{0,2}^{\alpha}(x) .
\end{aligned}
$$

EXAMPLE 3. Consider the system of generalized Abel's integral equation (1) with $u_{i, j}=1$ for $, j=1,2, n=1$ and $\mu=\frac{1}{3}$ with

$$
\begin{gathered}
h_{1}(x)=\frac{9}{40} x^{\frac{5}{3}}(4+3 x)+\frac{3}{440}(1-x)^{\frac{2}{3}}\left(40+9 x\left(5+6 x+9 x^{2}\right)\right) \\
h_{2}(x)=\frac{243}{440} x^{\frac{11}{3}}+\frac{3}{40}(1-x)^{\frac{2}{3}}(13+9 x(2+x)) .
\end{gathered}
$$

which has exact solutions $f_{1}(x)=x+x^{2}$ and $f_{2}(x)=x^{3}$. Apply the Gegenbauer wavelet approach and solve (14) by taking $M=$ $4, k=1$ and $\alpha>0$ we obtain exact solution

$$
\begin{aligned}
& x+x^{2}=\frac{\pi^{1 / 4}(7+6 \alpha) \sqrt{\frac{\Gamma\left[\frac{1}{2}+\alpha\right]}{\Gamma[1+\alpha]}}}{8 \sqrt{2}(1+\alpha)} \psi_{0,0}^{\alpha}(x)+\frac{\sqrt{\pi} \sqrt{\frac{4^{-\alpha} \Gamma[1+2 \alpha]}{(1+\alpha) \Gamma[\alpha]^{2}}}}{2 \alpha} \\
& \times \psi_{0,1}^{\alpha}(x)+\frac{\sqrt{\frac{\pi}{2}} \sqrt{\frac{2^{-2 \alpha}(2+2+2 \alpha]}{(2+\alpha) \Gamma[\alpha]^{2}}}}{8 \alpha+8 \alpha^{2}} \psi_{0,2}^{\alpha}(x), \\
& x^{3}=\frac{\pi^{1 / 4}(5+2 \alpha) \sqrt{\frac{\Gamma\left[\frac{1}{2}+\alpha\right]}{\Gamma[1+\alpha]}}}{16 \sqrt{2}(1+\alpha)} \psi_{0,0}^{\alpha}(x)+\frac{3 \sqrt{\pi}(5+2 \alpha) \sqrt{\frac{4^{-\alpha} \Gamma[1+2 \alpha]}{(1+\alpha) \Gamma[\alpha]^{2}}}}{32 \alpha(2+\alpha)} \\
& \times \psi_{0,1}^{\alpha}(x)+\frac{3 \alpha \Gamma\left[\frac{3}{2}+\alpha\right]}{8 \Gamma[3+\alpha] \sqrt{\frac{2^{1-2 \alpha} \Gamma[2+2 \alpha]}{(2+\alpha) \Gamma[\alpha]^{2}}}} \psi_{0,2}^{\alpha}(x) \\
& +\frac{\sqrt{3} \alpha(1+\alpha) \Gamma\left[\frac{3}{2}+\alpha\right]}{8 \Gamma[4+\alpha] \sqrt{\frac{2^{1-2 \alpha} \Gamma[3+2 \alpha]}{(3+\alpha) \Gamma[\alpha]^{2}}}} \psi_{0,3}^{\alpha}(x) .
\end{aligned}
$$

EXAMPLE 4. Consider the system of generalized Abel's integral equation (1) with $u_{1,1}=\frac{x^{2}}{2}, u_{1,2}=x, u_{2,1}=2-x, u_{2,2}=$ 6 for $n=2$ and $\mu=\frac{1}{2}$ with 
Table 1. Comparison between the approximate and exact solution of Example 1 for $M=6, k=1$ and $\alpha=1 / 2$

\begin{tabular}{|c|c|c|c|c|c|c|c|c|c|c|}
\hline $\mathrm{t}$ & 0 & 0.1 & 0.2 & 0.3 & 0.4 & 0.5 & 0.6 & 0.7 & 0.8 & 0.9 \\
\hline$f_{1}(x)$ & 1 & 1.0048 & 1.018 & 1.0408 & 1.070 & 1.106 & 1.148 & 1.196 & 1.249 & 1.306 \\
\hline$\tilde{f}_{1}(x)$ & 0.99 & 1.0048 & 1.018 & 1.040 & 1.070 & 1.106 & 1.148 & 1.196 & 1.249 & 1.306 \\
\hline$f_{2}(x)$ & 0 & 0.01 & 0.04 & 0.09 & 0.16 & 0.25 & 0.36 & 0.49 & 0.64 & 0.81 \\
\hline$\tilde{f}_{2}(x)$ & -0.00005 & 0.0099 & 0.0400 & 0.090 & 0.1600 & 0.2500 & 0.359 & 0.489 & 0.6400 & 0.81 \\
\hline
\end{tabular}

$$
\begin{aligned}
h_{1}(x) & =\frac{1}{8} \sqrt{\left(1-x^{2}\right)}\left(2+3 x^{2}\right)-3 x^{4} \log [x] \\
& +3 x^{4} \log \left[\sqrt{1-x^{2}}\right]+\frac{1}{8} x^{3}(4+\pi x)
\end{aligned}
$$

$h_{2}(x)=\frac{9}{8} \pi x^{4}+\frac{1}{2}(2-x)\left(3 \sqrt{1-x^{2}}+x^{2} \log \left[\frac{1+\sqrt{1-x^{2}}}{x}\right]\right.$.

which has exact solutions $f_{1}(x)=x+x^{2}$ and $f_{2}(x)=x^{4}$. If we apply the Gegenbauer wavelet approach and solve (14) by taking $M=5, k=1$ and $\alpha>0$ we obtain the exact solation

$$
\begin{aligned}
& x+x^{2}=\frac{\pi^{1 / 4}(7+6 \alpha) \sqrt{\frac{\Gamma\left[\frac{1}{2}+\alpha\right]}{\Gamma[1+\alpha]}}}{8 \sqrt{2}(1+\alpha)} \psi_{0,0}^{\alpha}(x)+\frac{\sqrt{\pi} \sqrt{\frac{4^{-\alpha} \Gamma[1+2 \alpha]}{(1+\alpha) \Gamma[\alpha]^{2}}}}{2 \alpha} \\
& \times \psi_{0,1}^{\alpha}(x)+\frac{\sqrt{\frac{\pi}{2}} \sqrt{\frac{2^{-2 \alpha} \Gamma[2+2 \alpha]}{(2+\alpha) \Gamma[\alpha]^{2}}}}{8 \alpha+8 \alpha^{2}} \psi_{0,2}^{\alpha}(x), \\
& x^{4}=\frac{2^{-\frac{1}{2}+2 \alpha} \sqrt{\frac{\Gamma\left[\frac{1}{2}+\alpha\right]}{\Gamma[1+\alpha]}} \Gamma[1+\alpha] \Gamma\left[\frac{9}{2}+\alpha\right]}{\pi^{1 / 4} \Gamma[5+2 \alpha]} \psi_{0,0}^{\alpha}(x) \\
& +\frac{\sqrt{\pi}(7+2 \alpha) \sqrt{\frac{4^{-\alpha} \Gamma[1+2 \alpha]}{(1+\alpha) \Gamma[\alpha]^{2}}}}{16 \alpha(2+\alpha)} \psi_{0,1}^{\alpha}(x) \\
& +\frac{3 \sqrt{\pi}(7+2 \alpha) \sqrt{\frac{2^{-1-2 \alpha} \Gamma[2+2 \alpha]}{(2+\alpha) \Gamma[\alpha]^{2}}}}{32 \alpha(1+\alpha)(3+\alpha)} \psi_{0,2}^{\alpha}(x) \\
& +\frac{\sqrt{3} \alpha(1+\alpha) \Gamma\left[\frac{3}{2}+\alpha\right]}{4 \Gamma[4+\alpha] \sqrt{\frac{2^{1-2 \alpha} \Gamma[3+2 \alpha]}{(3+\alpha) \Gamma[\alpha]^{2}}}} \psi_{0,3}^{\alpha}(x) \\
& +\frac{\sqrt{3} \alpha(1+\alpha) \Gamma\left[\frac{5}{2}+\alpha\right]}{8 \Gamma[5+\alpha] \sqrt{\frac{2^{1-2 \alpha} \Gamma[4+2 \alpha]}{(4+\alpha) \Gamma[\alpha]^{2}}}} \psi_{0,4}^{\alpha}(x)
\end{aligned}
$$

\section{Conclusion}

The aim of the present work is to develop an efficient and accurate method for solving system of generalized Abel's integral equation by using Gegenbauer wavelets in $L_{w^{\alpha}}^{2}(0,1]$ space. The $\mathrm{G}$ W reduces system of generalized Abel's integral equation to system of linear algebraic equations. Since number of basis functions are restricted up to seven so it does not make the calculation complexity. Illustrative examples are included to demonstrate the validity and applicability of the present technique.

\section{Acknowledgments}

The work of first author was supported by U.G.C Startup grant for newly recruited faculty.

\section{REFERENCES}

[1] M.Lowengrub , J. Waltson. System of generalizd Abel's integral equation. SIAM J. Math. Anal., 1979,10(4) : 794-807.

[2] J. Waltson. System of generalizd Abel's integral equation. SIAM J. Math. Anal., 1979,10(4) : 794-807.

[3] B. N. Mandal, A. Chakrabarti . Applied singular integral equations CRC Press, 2011.

[4] N.Mandal,A. Chakrabarti,B.N.Mandal Numerical solution of a system of generalized Abel's integral equation using fractional calculus. Appl. Math. Lett.,1996,9(5):1-4.

[5] Ashish Pathak, M. M. Dixit, P.S. Upreti ,Numerical solution of system of generalized Abel's integral equation using Legendre multiwavelet,J.Adv. Res. Rese.in Sci. comp.,2013, 5(1):28-36

[6] Ashish Pathak, Rajeev Kr. Singh, B.N. Mandal, Solution of Abel's integral equation by using Gegenbauer wavelets, Investigation in mathematical sciences, 2014,4(1), 43-52.

[7] I. Daubechies. Ten lectures on Wavelet . SIAM, Philadelphia, 1992.

[8] T. Fang, Li JQ, MN Sun. A universal solution for evolutionary random response problem. Journal of Sound Vibration 253(2002) 909-916. 